\title{
Tackling concussion in professional rugby union: a case-control study of tackle-based risk factors and recommendations for primary prevention
}

\author{
Matthew J Cross, ${ }^{1,2}$ Ross Tucker, ${ }^{3}$ Martin Raftery, ${ }^{3}$ Ben Hester, ${ }^{3}$ Sean Williams, ${ }^{2}{ }^{2}$ \\ Keith A Stokes, ${ }^{2}{ }^{2}$ raig Ranson, ${ }^{4,5}$ Prav Mathema, ${ }^{5}$ Simon Kemp ${ }^{1}$
}

\begin{abstract}
- Additional material is published online only. To view please visit the journal online (http://dx.doi.org/10.1136/ bjsports-2017-097912).
\end{abstract}

1Professional Rugby Department, Rugby Football Union, Twickenham, London, UK ${ }^{2}$ Department for Health, University of Bath, Bath, UK ${ }^{3}$ World Rugby, Dublin, Ireland ${ }^{4}$ Sports Injury Research Group, Cardiff Metropolitan University, Cardiff, UK

${ }^{5}$ Welsh Rugby Union, Cardiff, UK

Correspondence to

Matthew J Cross, Professional Rugby Department, Rugby

Football Union, Twickenham TW2 7BA, UK;

m.cross@bath.ac.uk

Accepted 12 September 2017 Published Online First 11 October 2017

Check for updates

To cite: Cross MJ, Tucker R, Raftery M, et al. Br J Sports Med 2019:53:1021-1025.

\section{ABSTRACT}

Background/aim Concussion, the most common injury in professional rugby union, occurs most commonly during the tackle. Thus, we investigated the association between tackle characteristics and concussion.

Methods 182 video clips of tackles leading to clinically diagnosed concussion and 4619 tackles that did not were coded across three professional rugby union competitions. A variable selection process was undertaken to identify the most important variables for interpretation. A multivariate generalised linear model was used to model the association between retained variables and concussion risk. Magnitude-based inferences provided an interpretation of the real-world relevance of the outcomes.

Results The four retained variables were: accelerating player, tackler speed, head contact type and tackle type. Overall, $70 \%$ of concussions occurred to the tackler and $30 \%$ to the ball carrier. There was a higher risk of concussion if the tackler accelerated into the tackle (OR: $2.49,95 \% \mathrm{Cl} 1.70$ to 3.64$)$ or the tackler was moving at high speed (OR: $2.64,95 \% \mathrm{Cl} 1.92$ to 3.63). Head contact with the opposing player's head (OR: 39.9, $95 \% \mathrm{Cl} 22.2$ to 71.1$)$ resulted in a substantially greater risk of concussion compared with all other head contact locations.

Conclusions Interventions that reduce the speed and acceleration of the tackler and reduce exposure to headto-head contact would likely reduce concussion risk in professional rugby union.

\section{INTRODUCTION}

Rugby union is a high-intensity collision sport with around 450 contact events per professional match, of which approximately 200 are tackles. ${ }^{1}$ Overall, concussions have been shown to account for almost one quarter of all reported time-loss match injuries during a professional season. ${ }^{2}$ This high frequency of occurrence in the professional game clearly makes the primary prevention of the injury a key priority for the sport.

Rugby union has introduced a significant number of concussion initiatives including law changes to support an off-field head injury assessment (HIA), the introduction of an operational definition of concussion $^{3}$ and mandatory stakeholder concussion education. These and other initiatives are thought to be key factors for the year-on-year increase in the reported incidence rate of match concussion within the English professional game over the period 2009-2016. ${ }^{2}$ Although much has been done to improve the awareness, identification and management of concussion in rugby, there has been little progress regarding primary prevention of the injury. ${ }^{4}$

The tackle is the most injurious match event in professional rugby union ${ }^{1}$ with high speeds, high tackle height and front on tackles increasing the risk of injury within the tackle. ${ }^{5-7}$ The majority of concussions occur in the tackle ${ }^{8}$ making it a focus for concussion prevention. However, the tackle characteristics associated with concussion in professional rugby union are not understood. In one recent study of 52 head impacts, tackler head placement and high-speed tackles were significant risk factors for head impact. ${ }^{9}$ However, whether the player was subsequently diagnosed with concussion was unknown. To our knowledge, no large-scale video analysis study investigating tackle-specific risk factors of time-loss concussion in rugby union exists. Thus, we investigated the association between tackle characteristics and clinically diagnosed concussion to inform future game-wide injury prevention initiatives.

\section{METHODS}

\section{Participants}

This case-control study was conducted in a population of 2029 first team male professional rugby players competing in three major professional rugby union competitions (The English Premiership, The Pro 12 and The Rugby World Cup). Data were collected over three seasons (2013/2014, 2014/2015 and 2015/2016). Written informed consent was obtained from each participant.

\section{Procedures}

A single experienced game analyst coded tackle events that led to a clinically diagnosed concussion (hereafter known as concussion), using a predefined coding matrix on a bespoke analysis platform (Fair Play Pty Ltd, Australia). For the purpose of this study, and in agreement with a previous study, ${ }^{5}$ the definition of a tackle used for the study was based on World Rugby law ${ }^{10}$ and was defined as 'any event where one or more tacklers attempted to stop or impede the ball carrier whether or not the ball carrier was brought to ground'.

Over the period of data collection, the HIA process was in place in order to aid both clinical decision making (in-game) and subsequent diagnosis (postgame) of concussion. This three-point time assessment process ${ }^{3}$ was consistent over the 
study period and was the operational process that informed the clinical diagnosis of concussion made by the team doctors in this study. Concussion cases that occurred in domestic competition were reported in each union's respective, well-established injury surveillance system. Similarly, concussions that occurred during the Rugby World Cup (RWC) 2015 were reported via the RWC injury surveillance system. All three surveillance systems used consistent definitions and were aligned with the consensus statement for the reporting of injuries in rugby union. ${ }^{11}$ These systems reported detailed injury information (such as injury date, competition, playing position, injury event and time of injury) to help identify the specific match event that led to the player being diagnosed with concussion. Any concussions reported as delayed or evolving were not included in the study as they could not be directly associated to a specific match event.

The coding matrix applied to each video clip comprised 15 categorical variables, the majority of which described characteristics of the tackle but also included pretackle characteristics (such as player position and preceding event). The coding matrix was developed from the templates used in previous studies investigating tackle injuries in professional rugby union. ${ }^{5-7}$ The variables reported in this study were: identification of the accelerating player, tackler speed, contact type and tackle type. The inclusion of these variables specifically was determined by the modelled degree of importance of each variable (see data analysis section below).

In addition, a control group of 4619 tackles that did not result in a concussion were coded from 28 matches in representative professional rugby competitions. These were coded by the same analyst to calculate frequency of occurrence in normal match play and to help quantify the value of any potential recommendations for the game on concussion risk. Video clips were excluded if: (A) the quality of the video footage did not allow the match event to be clearly observed or (B) the footage was of insufficient quality to apply the coding template to the tackle.

\section{Data analysis}

All estimations were made using $R$ (V.3.3.1, R Foundation for Statistical Computing, Vienna, Austria). First, in order to establish the importance of each coded variable in predicting the risk of concussion in the tackle, a machine learning model was used to identify the variables that, when removed from the model, were associated with the largest increase in concussion prediction error (ie, the variables that were associated with the biggest decrease in predictive accuracy when they were not included). This was performed using the variable importance feature within the randomForest package, ${ }^{12}$ with an increase in mean square error (MSE) of 20\% set as a threshold for retaining variables. ${ }^{13}$ This then also allowed us to select the most parsimonious model for further statistical analysis. ${ }^{14}$ Subsequently, a multivariate generalised linear model (GLM), with binomial distribution and logit link, was used to model the associations between the different scenarios within each retained variable and risk of concussion. Correlation coefficients between the variables, alongside variance inflation factors (VIFs), were used to detect multicollinearity between the predictor variables. A VIF of $\geq 10$ was deemed indicative of substantial multicollinearity. ${ }^{15}$ The most frequently occurring category within each variable was used as the reference condition. The resultant ORs were plotted against the overall frequency of each tackle characteristic, to allow interpretation of both the risk associated with the given tackle characteristic and the regularity with which it occurs. ${ }^{16}$

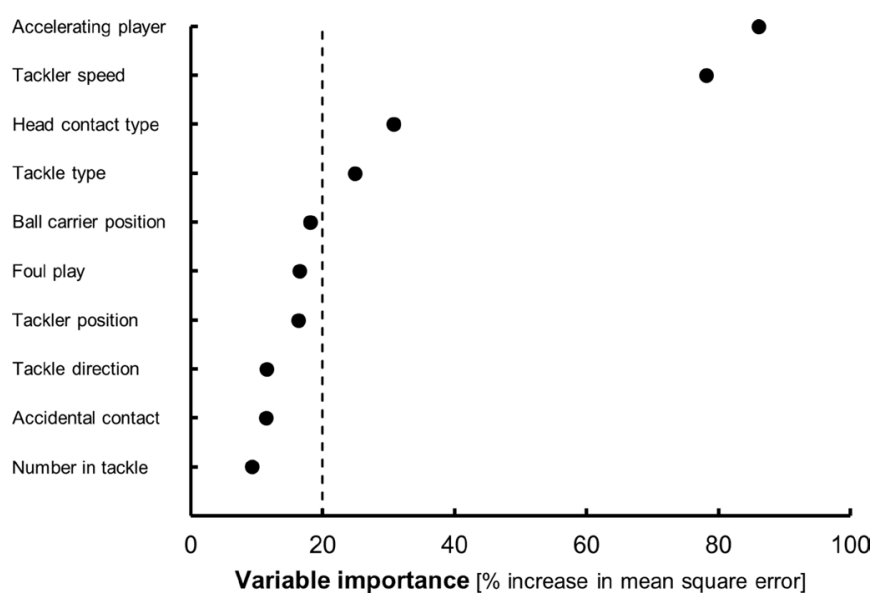

Figure 1 The increase in model error (decrease in the ability of the model to predict concussion) when each variable is removed. Dashed line highlights $20 \%$ threshold for variable retention.

The associated 95\% CIs were used to represent the likely range of the true value.

Magnitude-based inferences were used to provide an interpretation of the real-world relevance of the outcomes. ${ }^{17}$ The smallest important increase in injury risk was a relative risk of 1.11 , and the smallest important decrease in risk was $0.90 .^{18}$ Effects were classified as unclear if the percentage likelihood that the true effect crossed both positive and negative smallest worthwhile effect thresholds were both greater than 5\%. Otherwise, the effect was deemed clear and was qualified with a probabilistic term using the following scale: $<0.5 \%$ : most unlikely; 0.5\%-5\%: very unlikely; 5\%-25\%: unlikely; 25\%-75\%: possible; 75\%-95\%: likely; 95\%-99.5\%: very likely; >99.5\%: most likely. ${ }^{19}$

\section{RESULTS}

Two hundred and forty-seven concussions where the player was permanently removed from play were identified through interrogation of the three primary injury surveillance systems. Sixty-five of these were excluded due to either (A) insufficient video quality or (B) not being able to identify the causal event in the video. This left 182 concussions that were sustained in 171 matches available for further analysis in this study. Of the 182 concussions, $70 \%$ of concussions were sustained to the tackler and $30 \%$ to the ball carrier.

Within the statistical model, the most important variables (ranked from highest to lowest) for predicting concussion outcome were: accelerating player (percentage increase in MSE when the variable was removed: $86 \%)$, tackler speed (78\%), head contact type (31\%) and tackle type (25\%) (figure 1). Within the accelerating player variable, there was a most likely higher risk of concussion if the tackler was accelerating into the tackle (OR: $2.49,95 \%$ CI 1.70 to 3.64 ) and a likely higher risk when both players accelerate into the tackle (OR: 1.44, 95\% CI 1.01 to 2.04) when compared with an accelerating ball carrier (figure 2). Similarly, when the tackler was moving at high speed, there was a most likely higher risk of concussion (OR: 2.64, 95\% CI 1.92 to $3.63)$ when compared with the reference scenario of low speed (figure 3).

Head contact with the opposing player's head (OR: 39.9, 95\% CI 22.2 to 71.1 ), ground (OR: $21.8,95 \%$ CI 7.8 to 61.3 ) and knee (OR: $20.395 \%$ CI 10.2 to 40.3 ) had substantially greater risk of concussion compared with all other locations. 
Accelerating player

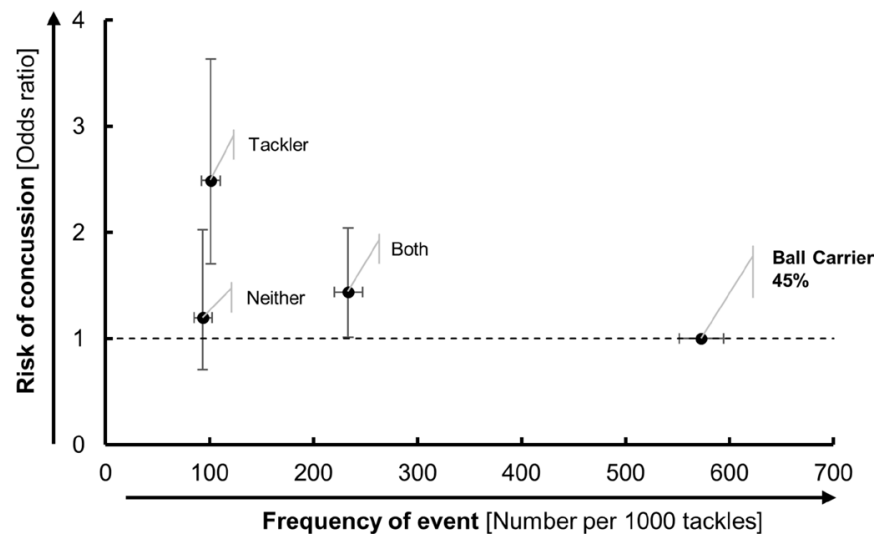

Figure 2 Accelerating player: likelihood of concussion for each given subvariable when compared with the most common scenario of ball carrier acceleration. X-axis shows frequency of occurrence as number per 1000 tackles. The within variable proportion of concussions attributed to the most common subvariable is also shown (\%).

However, these scenarios occurred infrequently compared with more common impact types such as head to trunk, lower limb and pelvis impacts (figure 4). All subvariables analysed were more likely to result in concussion than the most common and lowest risk scenario of head to trunk contact (figure 4). All illegal tackle types (referee determined high tackle, tip tackle and tackle in the air) were associated with the highest risks of concussion, but all were rare events (these tackle types occur at a frequency of $\leq 2$ per 1000 tackles). High tackles were 36.5 times more likely (OR: 36.5 , 95\% CI 24.7 to 53.9 ) to result in a concussion when compared with passive shoulder tackles (figure 5).

\section{DISCUSSION}

In our study of tackle characteristics and concussion, 70\% of concussions associated with the tackle were sustained to the tackler and $30 \%$ to the ball carrier. In order to establish the importance of each coded variable in predicting the risk of concussion in the tackle, we pioneered using a machine

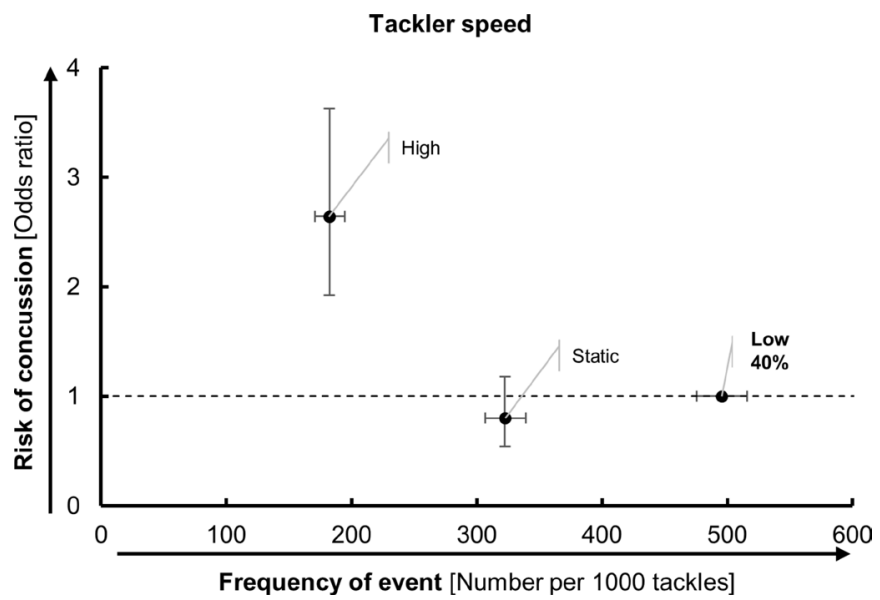

Figure 3 Tackler speed: likelihood of concussion for each given subvariable when compared with the most common scenario of a low speed tackler. X-axis shows frequency of occurrence as number per 1000 tackles. The within variable proportion of concussions attributed to the most common subvariable is also shown (\%).

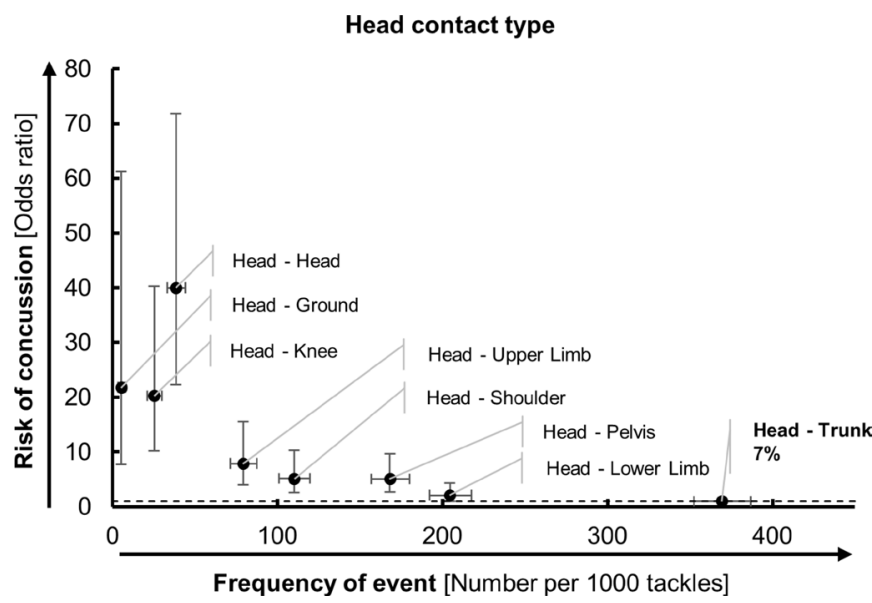

Figure 4 Contact type: likelihood of concussion for each given subvariable when compared with the most common scenario of a headtrunk contact. $X$-axis shows frequency of occurrence as number per 1000 tackles. The within variable proportion of concussions attributed to the most common subvariable is also shown (\%).

learning model to identify the variables that, when removed from the model, were associated with the largest increase in concussion prediction error. The (1) accelerating player, (2) tackler speed, (3) head contact type and (4) tackle type were identified as the four tackle characteristics that statistically represented the greatest likelihood for modifying the risk of concussion within a tackle. Accelerating player and tackler speed variables had the largest influence on whether a concussion occurred in the tackle.

Next, a multivariate GLM was used to model the associations between the different scenarios within each retained variable and the likelihood of concussion. Specifically, the likelihood of concussion increased significantly if (A) the tackler or both players were accelerating rather than the ball carrier accelerating, (B) the tackler was at high speed rather than at low speed or (C) if the head made contact with the opposing player's head, knee or the ground rather than the most common location (the trunk). Furthermore, high tackles were 36.5 times more likely to result in a concussion compared with passive shoulder tackles. Of the

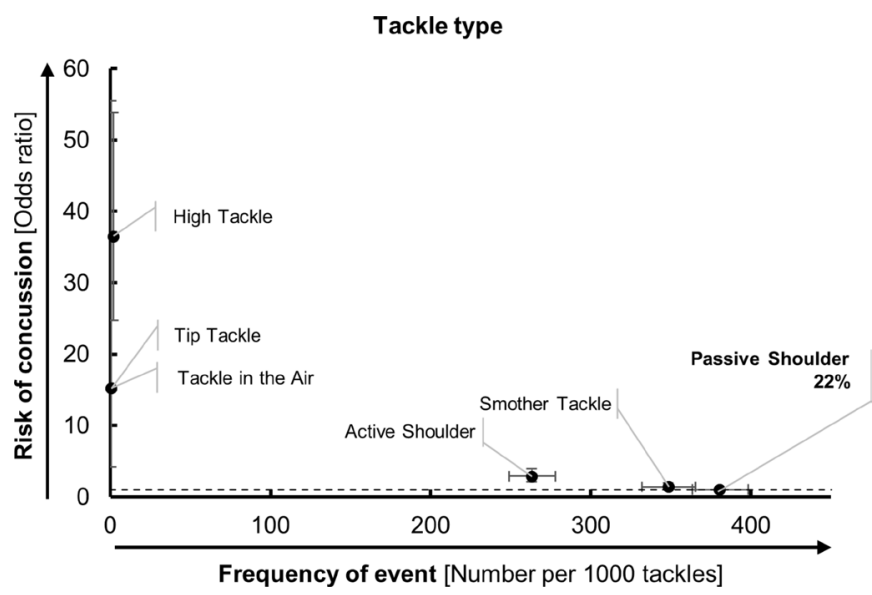

Figure 5 Tackle type: likelihood of concussion for each given subvariable when compared with the most common scenario of passive shoulder tackles. X-axis shows frequency of occurrence as number per 1000 tackles. The within variable proportion of concussions attributed to the most common subvariable is also shown (\%). 
variables analysed, the subvariables associated with the highest risk of concussion were never the most frequent, suggesting that the current structure and governance of the game already goes some way to reducing player exposure to the highest risk tackle characteristics.

\section{Concussion prevention opportunities}

To successfully reduce the risk of concussion in professional rugby union, the theoretical options for risk mitigation need to be considered against the real-world landscape. ${ }^{20}$ We identified the tackle characteristics that were most associated with the risk of concussion and we noted their frequency. The frequency of each event is important as a moderate risk; high frequency event may cause more concussions than a high-risk, low-frequency event. That is not to say, for example, that reducing the frequency of high risk but relatively uncommon events such as referee-determined high tackles should be overlooked. This should still be a target to reduce concussion risk, but it may not yield the same real-world benefit as a risk reduction strategy focused on reducing the frequency of a more common match scenario.

The most effective, although extreme, method for preventing concussion would be to eliminate exposure by removing the tackle from the game. ${ }^{21}$ However, removing one of the sports integral game events would change rugby union beyond recognition $^{21} 22$ and is therefore unlikely to be a practicable option at the professional level. A more considered approach would be to suggest modifications to, or the reinforcement of, existing law to drive changes in player behaviour. ${ }^{4}$ This is a model that has been credited with significant success in other areas of the game such as the scrum ${ }^{23}$ and in other contact sports such as ice hockey. ${ }^{24}$ In addition, initiatives that focus on addressing the technical aspects of the tackle via coach and player education could also be beneficial in reducing concussion risk if structured, translated and implemented appropriately. ${ }^{20}$ The potential of this approach is discussed in more detail later.

\section{Tackler speed and acceleration}

The most compelling finding from this study was the association between tackler movement (both acceleration and speed) and the risk of concussion. Previous studies have also suggested that the velocity dynamics of the tackle increase the risk of injury, ${ }^{17}$ but we have specifically identified an association between the tackler's movement profile and concussion risk. The most commonly observed game situation that led to a high speed and/ or accelerating tackler was where the tackling player 'rushed' up out of the defensive line to make an effective tackle and specifically attempted to try and stop the ball carrier making the pass.

Limiting the speed of the tackler in the performance-focused professional game will likely prove a complex challenge, but based on the findings of this study, will likely afford the biggest reduction in concussion risk (but not necessarily incidence given the low frequency of occurrence of these events). When the tackler was travelling at high speed, more injuries were sustained by backs than forwards (data not shown). Thus, one strategy for consideration might be to reduce the space between 'backlines' at set piece plays to reduce subsequent energy transfer ${ }^{7}$ in the tackle. The potential negative consequence of this may however be that tacklers accelerate into tackles more often to attain physical dominance in the contact event. Presently, that situation is relatively rare, but an increase in frequency of tacklers accelerating would in fact increase concussion risk, as we have shown in the results of this study.

\section{Tackler versus ball carrier risk}

In agreement with previous research conducted in elite rugby union, ${ }^{9}$ the majority of concussions in this study were sustained to the tackler (70\%). Moreover, recent data from the English Premiership showed that $46 \%$ of all injuries sustained to the tackler during a competitive season were concussion, ${ }^{2}$ further highlighting the need to focus on reducing the number of concussions sustained specifically by the tackler.

The fact that there is a difference in the risk of concussion between the tackler and ball carrier is unsurprising given that their direction and points of application of energy in the tackle differ. $^{7}$ The existing tackle law limits the height of the tackler's contact on the ball carrier to the line of the ball carrier's shoulders, thereby reducing the risk of contact with the head of the ball carrier. Consistent application of this law with sanctions for non-compliance by the tackler is critical in minimising the risk of concussion to the ball carrier. However, it is conceivable that further modification (lowering) of the permitted height of the tackler's contact with the ball carrier may reduce the risk of head injury to both players, with relatively greater reductions in risk to the ball carrier. ${ }^{6}$ It is important to consider that the tackler, who is at greatest risk of head injury even for higher impact types such as head-to-head contact, may also be protected to some extent by the lowering of tackle height, with the potential to reduce the absolute number of concussions by a meaningful amount, although the magnitude of this relationship remains unknown.

It is therefore probable that to directly increase the safety of the tackler, the most efficacious approach would likely be to focus on creating technical and/or tactical modifications through coach and player awareness and education. A recent study in elite youth players demonstrated an association between tackle technique and concussion, ${ }^{25}$ and although not formally studied, cases of poor tackle technique leading to injury have been observed in the professional game. ${ }^{7}$ In addition, a biomechanical analysis of 15 rugby union players suggested that tackles executed on the non-dominant side were less compliant with current coaching recommendations regarding head position. ${ }^{26} \mathrm{~A}$ large case-control study in the professional game would likely provide insight into the specific areas of focus for such player safety initiatives in the future. It is acknowledged that it remains unknown as to whether this type of intervention would be more or less effective in the professional game compared with the amateur game, and further research is required to guide the judicious application of such an intervention.

\section{Head contact}

Referee-determined high tackles were 36.5 times more likely to result in a concussion when compared with the most common tackle type (passive shoulder), and in addition, head-to-head contacts were around 40 times more likely to result in a concussion when compared with head-to-trunk contact. Both of these findings are in agreement with findings presented in previous studies. ${ }^{5-7}$ It is of note that these findings also support World Rugby's recent initiative of a zero-tolerance towards head contact with game-wide increased sanctions now in operation for contact with the head. ${ }^{27}$ However, while efforts to shift actions away from the highest risk events should be applauded, it is also important to monitor the possibility that reducing risk in one area of the game may change the risk in another. ${ }^{28}$ In this study, head-to-knee and head-to-ground contacts were also high risk, with approximately a 20 -fold increase in risk when compared with the most common head contact type of 
head to trunk. However, any intervention that replaces headto-head impacts with any other impact type stands to reduce the incidence of concussion based on the present data. One previous study found that the all-injury risk to the tackler increased when the tackler made low tackles. ${ }^{7}$ However, it is important to note that lowering the tackle height would not necessarily increase the frequency of low tackles per se. As mentioned, the desired outcome would be to increase the frequency of mid-height tackles where the risk of concussion is lowest (figure 4). Since head-to-trunk contact types are already the most frequently occurring, it would seem likely that any interventions designed to minimise contact with the head may increase the frequency of these tackle types. This would, as a consequence, reduce the incidence of concussion in the professional game. Due to the unpredictability of possible outcomes following any tackle-height law intervention, the continued monitoring of all injury risk using well-established injury surveillance systems is warranted. It should also be noted that the illegal tackle types (referee determined high tackle, tip tackles and tackles in the air) were the rarest events suggesting that current law is effective in deterring these behaviours.

\section{Limitations}

One limitation of this study was the reliance on subjective interpretation for many of the tackle variables presented. In future, microtechnology could be used to quantify data such as relative speeds and thus improve the objectivity of the analysis. Separately, a number of concussions were excluded from the analysis due to poor video footage or postmatch presentation. These cases, particularly those with delayed onset of symptoms, may differ from those presenting on field, and the present study is unable to examine this possibility. While illegal tackle types were identified based on referee decisions in the study (high tackle, tip tackle and so on), whether the law was applied correctly in each situation (ie, did the referee make the correct decisions at the time) remains unknown and warrants further investigation. While adequately powered to detect subtle differences in concussion risk between variables in our multivariate model, a number of these variables were subject to sparse data bias and should be interpreted with caution (online supplementary files). ${ }^{29}$ In addition, this study was underpowered for the analysis of subvariable interactions and to consider the effect of different tackle characteristics on injury severity. Given the likely differences

\section{What are the findings?}

- The majority of tackle-related concussions are sustained by the tackler.

- Concussion risk was greater if the tackler accelerated into the tackle or moved at high speed.

- Concussion risk was greater when there was head contact with the opposing player's head or knee, or head contact with the ground.

\section{How might it impact on clinical practice in the future?}

This paper provides evidence and direction for national and international governing bodies to explore coaching and rule changes to methods for reducing the speed and acceleration of the tackler and for reducing the occurrence of head-to-head contact. in physical and tactical game characteristics at different levels of the rugby, these findings are unlikely to be generalisable to non-professional cohorts.

\section{Summary and conclusions}

Tackles that result in head-to-head contact have the high risk of concussion. Change, reinforcement and/or modification of existing tackle law particularly regarding the height of the tackle may reduce direct contact with the head of the ball carrier. Furthermore, identifying ways of reducing the speed and acceleration of the tackler while they make effective tackles must also explored. We emphasise that rule changes bring with them unknown impact on the risk of other injury types.

\section{Twitter Matt Cross @mattjcrossie}

Contributors MJC, SK, MR and RT conceived and designed the study and coding template. BH coded all video clips for analysis, and MJC, SW and KAS analysed and interpreted the data. MJC prepared the first draft of the manuscript and then all authors made substantial contributions to the various iterations of the manuscript resulting in the final version.

Competing interests MJC and SK are employed by the Rugby Football Union. MR, BH and RT are employed by World Rugby. CR and PM are employed by the Welsh Rugby Union and have previously received research funding from World Rugby. SW and KAS are employed by the University of Bath and have previously received research funding from the Rugby Football Union.

Ethics approval Research Ethics Approval Committee for Health (REACH) at the University of Bath.

Provenance and peer review Not commissioned; externally peer reviewed.

(C) Article author(s) (or their employer(s) unless otherwise stated in the text of the article) 2019. All rights reserved. No commercial use is permitted unless otherwise expressly granted.

\section{REFERENCES}

1 Fuller CW, Brooks JH, Cancea RJ, et al. Contact events in rugby union and their propensity to cause injury. Br J Sports Med 2007:41:862-7.

2 Rugby Football Union. The professional rugby injury surveillance project: the 2015-16 annual report. 2017.

3 Raftery M, Kemp S, Patricios J, et al. It is time to give concussion an operational definition: a 3-step process to diagnose (or rule out) concussion within $48 \mathrm{~h}$ of injury: World Rugby guideline. Br J Sports Med 2016;50:642-3.

4 Batten J, White AJ, Anderson E, et al. From management to prevention: the new cure for sports concussion. Br J Sports Med 2016:1293-4.

5 Fuller CW, Ashton T, Brooks JH, et al. Injury risks associated with tackling in rugby union. Br J Sports Med 2010;44:159-67.

6 Mclntosh AS, Savage TN, McCrory P, et al. Tackle characteristics and injury in a cross section of rugby union football. Med Sci Sports Exerc 2010;42:977-84.

7 Quarrie KL, Hopkins WG. Tackle injuries in professional Rugby Union. Am J Sports Med 2008;36:1705-16.

8 Cross M, Kemp S, Smith A, et al. Professional Rugby Union players have a $60 \%$ greater risk of time loss injury after concussion: a 2-season prospective study of clinical outcomes. Br J Sports Med 2016:50.

9 Tierney GJ, Lawler J, Denvir K, et al. Risks associated with significant head impact events in elite Rugby Union. Brain Inj 2016;30:1350-61.

10 World Rugby. Law 15 - tackle definition. 2009 http://laws.worldrugby.org/?law=15\& language $=$ EN (accessed 5 Feb 2017).

11 Fuller CW, Molloy MG, Bagate C, et al. Consensus statement on injury definitions and data collection procedures for studies of injuries in Rugby Union. Br J Sports Med 2007;41:328-31.

12 Liaw A, Wiener M. Classification and regression by randomForest. $R$ news 2002;2:18-22

13 Li H, Leung KS, Wong MH, et al. Substituting random forest for multiple linear regression improves binding affinity prediction of scoring functions: cyscore as a case study. BMC Bioinformatics 2014;15:291

14 Genuer R, Poggi J-M, Tuleau-Malot C. Variable selection using random forests. Pattern Recognit Lett 2010;31:2225-36.

15 Kutner MH, Nachtsheim C, Neter J. Applied linear regression models. New York, USA: McGraw-Hill, 2004.

16 Lachance J. Disease-associated alleles in genome-wide association studies are enriched for derived low frequency alleles relative to HapMap and neutral expectations. BMC Med Genomics 2010;3:57.

17 Batterham AM, Hopkins WG. Making meaningful inferences about magnitudes. Int $J$ Sports Physiol Perform 2006;1:50-7. 


\section{Original article}

18 Hopkins WG. Linear models and effect magnitudes for research, clinical and practical applications. SportSci 2010;14:49-58.

19 Hopkins WG. A spreadsheet for deriving a confidence interval, mechanistic inference and clinical inference from a p value. SportSci 2007;11:16-20.

20 Finch C. A new framework for research leading to sports injury prevention. J Sci Med Sport 2006;9:3-9.

21 Fuller CW. Managing the risk of injury in sport. Clin I Sport Med 2007:17:182-7.

22 Tucker R, Raftery M, Verhagen E. Injury risk and a tackle ban in youth Rugby Union: reviewing the evidence and searching for targeted, effective interventions. A critical review. Br J Sports Med 2016;50:921-5.

23 Preatoni E, Cazzola D, Stokes KA, et al. Pre-binding prior to full engagement improves loading conditions for front-row players in contested rugby Union scrums. Scand J Med Sci Sports 2016;26:1398-407.
24 Black AM, Macpherson AK, Hagel BE, et al. Policy change eliminating body checking in non-elite ice hockey leads to a threefold reduction in injury and concussion risk in 11- and 12-year-old players. Br J Sports Med 2016;50:55-61.

25 Hendricks S, O'connor S, Lambert M, et al. Contact technique and concussions in the South African under-18 Coca-Cola craven week rugby tournament. Eur J Sport Sci 2015;15:557-64

26 Seminati E, Cazzola D, Preatoni E, et al. Tackle direction and dominant side affect upper body loading during rugby tackles. Br J Sports Med 2017;51:386.1-386.

27 World Rugby. New tackle laws to limit contact with the head announced. $2017 \mathrm{http}: / /$ www.worldrugby.org/news/213339? lang=en (accessed 21 Feb 2017).

28 Hagel B, Meeuwisse W. Risk compensation: a "side effect" of sport injury prevention?. LWW, 2004.

29 Greenland S, Mansournia MA, Altman DG. Sparse data bias: a problem hiding in plain sight. BMJ 2016;352:i1981. 